\title{
Le mariage dans l'Europe des $\mathrm{XVI}^{\mathrm{e}}$ et XVII siècles:
} réalités et représentations, textes réunis par Richard Crescenzo, Marie Roig-Miranda et Véronique Zaercher

\section{Gabriella Bosco]}

\section{(2) OpenEdition}

\section{Journals}

Edizione digitale

URL: https://journals.openedition.org/studifrancesi/38626

DOI: $10.4000 /$ studifrancesi.38626

ISSN: 2421-5856

Editore

Rosenberg \& Sellier

Edizione cartacea

Data di pubblicazione: 15 décembre 2004

Paginazione: 681-682

ISSN: 0039-2944

\section{Notizia bibliografica digitale}

Gabriella Bosco], «Le mariage dans l'Europe des XVIe et XVIIe siècles: réalités et représentations, textes réunis par Richard Crescenzo, Marie Roig-Miranda et Véronique Zaercher», Studi Francesi [Online], 144 (XLVIII | III) | 2004, online dal 30 novembre 2015, consultato il 08 mai 2021. URL: http:// journals.openedition.org/studifrancesi/38626 ; DOI: https://doi.org/10.4000/studifrancesi.38626

Questo documento è stato generato automaticamente il 8 mai 2021.

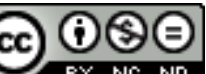

Studi Francesi è distribuita con Licenza Creative Commons Attribuzione - Non commerciale - Non opere derivate 4.0 Internazionale. 
Le mariage dans l'Europe des XVI et $X V I I^{\mathrm{e}}$ siècles: réalités et représentations, textes réunis par Richard Crescenzo, Marie Roig-Miranda et Véronique Zaercher

\author{
Gabriella Bosco]
}

\title{
NOTIZIA
}

AA. VV., Le mariage dans l'Europe des $\mathrm{XVI}^{\mathrm{e}}$ et XVII ${ }^{\mathrm{e}}$ siècles: réalités et représentations, textes réunis par Richard Crescenzo, Marie Roig-Miranda et Véronique Zaercher, Université Nancy II, 2003, 2 voll., I, pp. 375, II, pp. 280.

1 Atti di un convegno tenutosi a Nancy nel novembre 2001, i due volumi a cura di Richard CRESCENZO, Marie ROIG-MIRANDA e Véronique ZAERCHER indagano la questione matrimoniale da un duplice punto di vista: l'istituzione reale e le sue trasposizioni letterarie e artistiche. Nel primo ambito, i contributi passano in rassegna le dimensioni giuridica, canonica e politico-economica del matrimonio, le sue influenze sul divenire storico attraverso le alliances tra case regnanti, e, d'altro canto, le riflessioni d'ordine filosofico e teologico che l'istituto matrimoniale suscita, da parte di chi lo approva e da parte di chi lo contesta. Nell'ambito delle trasposizioni letterarie risulta evidente dall'insieme degli articoli ad esse dedicati - come la cifra dominante sia quella satirica. Ne viene fuori un ritratto del matrimonio in Europa tra Cinquecento e Seicento come di una faccenda estremamente serie per gli adepti, totalmente faceta per gli osservatori.

2 Il primo dei due volumi è maggiormente centrato sull'aspetto serio. Diviso in quattro sezioni, parte dalle basi giuridiche del matrimonio (Estrella RUIZ-GÀLVEZ PRIEGO, "Divinas Palabras: le libre consentement et le droit formulaire dans le contract/sacrement, 
Espagne, $\mathrm{XV}^{\mathrm{e}}-\mathrm{XVII}{ }^{\mathrm{e}}$ siècles"; Jean GAY, "Le mariage face aux règles juridiques et à l'organisation sociale dans la France coutumière aux XVI-XVII ${ }^{\mathrm{e}}$ siècles"), passa poi ad analizzare il matrimonio come fondamento dell'ordine economico e dell'identità sociale e nazionale con un approccio molto sfaccettato: il matrimonio dei turchi e la sua rappresentazione nei racconti di viaggio del XVI secolo, Marie-Christine GOMEZ-GÉRAUD; il matrimonio ebraico, Janine STRAUSS; l'atteggiamento della chiesa cattolica e il matrimonio degli indiani del Perù, Nicole FOURTANÉ; il matrimonio musulmano nel teatro del secolo d'oro, Yvette CARDAILLAC-HERMosilla; e l'esplosione della cellula familiare in seguito alla cacciata dei Mori da Granada dopo il drammatico sollevamento degli Alpujarras, all'inizio del XVII secolo, Catherine GAIGNARD.

3 La terza sezione riguarda i matrimoni d'alto rango, più che altro mercimoni, di potere $\mathrm{e}$ denaro, anche in questo caso visti come fatti storici e come oggetti letterari (dal matrimonio politico al matrimonio d'amore, Louis CARDAILLAC; il matrimonio segreto di Inès de Castro, Adrien RoIG; i canti pastorali di Ronsard sui matrimoni principeschi, del 1559, Yvonne BELLENGER; l'annullamento, alla corte del Portogallo, del matrimonio tra il re D. Afonso VI e Marie-Françoise di Savoia, 1668, Michel GUIRAUD; propaganda e ragion di stato nelle alliances reali franco-spagnole del XVII secolo, Alexandra TESTINOzAFIRopoulos; e, da Molière a Madame de Sévigné, l'importanza del denaro nei fatti matrimoniali, Roger DUCHÊNE). La quarta ed ultima sezione analizza lo scarto tra visione reale e realtà, nell'ottica del padre Francisco de Osuna (Jacqueline FERRERAS), di Ariosto e Tasso (Jean LACROIX), di Antonio López de Vega (Marie-Laure ACQUIER), di Cervantes (Laurence KOHN-PIREAUX), di Mateo Alemàn (Monique MICHAUD) nelle novelle del XVI secolo (Khédija AJROUD), nel romanzo bizantino-spagnolo (Christine MARGUET), nel romanzo francese della prima metà del XVII secolo, in particolare nell'Astrée (Laurence PlAZENETtE) e ne La Prétieuse di Michel de Pure (Myriam MÂITRE).

Il secondo volume è riservato più ai vizi che alle virtù del matrimonio. La prima sezione, è vero, si sofferma sulla visione di Erasmo (Louis LOBBES), di Montaigne e P.C. Hooft (Wim J. A. вотs), sulla rappresentazione della perfetta sposa, moglie innamorata, nella letteratura spagnola di Cinquecento e Seicento (Marie-Veronique MARTINEZ), sul discorso prescrittivo in Francia tra XVI e XVII secolo (Guy SPIELMANN), sul letto coniugale come tempio nel matrimonio riformato (Catherine MOINS, alla cui memoria gli atti sono dedicati, essendo scomparsa poco dopo aver partecipato al convegno), e sull'idealizzazione del metrimonio nel teatro francese tra fine Seicento e inizio Settecento (Jean-Philippe GROSPERRIN). Ma la sezione successiva tratta della trasgressione: relazioni extra matrimoniali, clandestinità, separazione. Virginie DUMANOIR si occupa dei romances di primo Cinquecento, Louis IMPERIALE di Mélibée e Lozana, Marie RoIG-MIRANDA del matrimonio come punizione in Tirso de Molina, André FENRANDEZ di bigamia tra il 1560 e il 1700, Mary-Nelly FOULIGNY di matrimonio e stregoneria, Alicia OÏFFER-BOMSEL di divorzio e separazione dopo il Concilio di Trento, e Claire CARLIN di adulterio secondo i trattati cattolici secenteschi. L'ultima sezione, poi, è un fuoco d'artificio sulle rappresentazioni satiriche del matrimonio: in Philippe de Vigneulles (Pierre DEMAROLLE), in Rabelais (Veronique ZAERCHER), da parte di pittori e incisori rinascimentali (Martine VASSELINE) e dei poeti satirici francesi nella prima metà del Seicento (Gilles BANDERIER), così come nelle plaidoiries cinquecentesche (Lyndan WARNER), e nell'opera di D. Francisco Manuel de Melo, scrittore luso-castigliano d'inizio Seicento che s'ispira a Pietro Aretino (Mariana GoIS NEVES). 